\title{
Joints Parameters Identification in Numerical Modeling of Structural Dynamics
}

\author{
Yuedong Yang, ${ }^{1,2}$ Jiqing Chen $\mathbb{D}^{1,}{ }^{1,2}$ Fengchong Lan, ${ }^{1,2}$ Fei Xiong, ${ }^{3}$ and Zicong Zeng ${ }^{1,2}$ \\ ${ }^{1}$ School of Mechanical \& Automotive Engineering, South China University of Technology, Guangzhou 510640, China \\ ${ }^{2}$ Guangdong Province Key Laboratory of Automotive Engineering, Guangzhou 510640, China \\ ${ }^{3}$ Guangzhou Automobile Group Co. Ltd. Automotive Engineer Institute, Guangzhou 511434, China \\ Correspondence should be addressed to Jiqing Chen; chjq1301@hotmail.com
}

Received 16 January 2018; Revised 29 March 2018; Accepted 2 May 2018; Published 5 June 2018

Academic Editor: Marcello Vanali

Copyright (C) 2018 Yuedong Yang et al. This is an open access article distributed under the Creative Commons Attribution License, which permits unrestricted use, distribution, and reproduction in any medium, provided the original work is properly cited.

\begin{abstract}
The dynamics of assembled structures are significantly dependent on joints. Joints parameters, owing to be difficultly measured, are always ignored by pure rigid in numerical modeling and it will result in unreliable or even error descriptions. $1 \sim 3 \mathrm{~Hz}$ deviations may cause resonance especially in engineering optimization issues; hence it is necessary to identify joints parameters for structural dynamics investigation. In present work, multiobjective optimization algorithms are used to identify joints parameters by approaching actual test results in each series of structure to decrease unreliable or error for numerical modeling. Taking automotive dynamics with seam-welding and spot-welding as examples, the relationship of joints parameters perturbation and structural dynamics is derived to give the selecting reason of parameters' identification. The actual dynamics of an SUV's frame and a thinwalled part in BIW (body in white) are utilized to validate the methodology. Results demonstrate that the validated model has enough accuracy to reflect the dynamics of the actual structure. The methodology provides reliable guarantee for dynamic analysis and the design of structure.
\end{abstract}

\section{Introduction}

All assembled structures are joined to each other through different types of joints, such as welds, rivets, and bolts [1]. Mechanical joints have significant influences on modal parameters, and the dynamic behavior of the assembled structure is highly dependent on joints [2]. In order to have an accurate understanding of the dynamics of an assembled structure, joints properties need to be accurately identified [1]. Structural joints as local characteristics are always ignored as pure rigid in simulations [3], which will result in unreliable predictions and even error. Finite element (FE) predictions are often called into question when they are in conflict with test results [4]. Inaccuracies in FE model and errors in results predicted by them can arise due to the use of incorrect modeling of boundary conditions, incorrect modeling of joints (degree of flexibility varies), and difficulties in modeling of damping. Hence, accurate mathematical models are required to describe the vibration characteristics of structures, which subsequently can be used for design purposes to limit the negative effects of vibrations [5].

Bograd et al. [6] reviewed different methods for modeling the dynamic behavior of mechanical joints in assembled structures. In practice, the validation of finite element dynamic models was mainly through comparing experimental modal analysis results with analytical predictions [7]. Joint parameters could be experimentally determined by the frequency response functions measured, respectively, with and without the joints [8-14]. However, only use of natural frequencies in a model updating process is probably advantageous for a couple of reasons: (1) natural frequencies are usually more sensitive to the variations of model parameters, and (2) they are normally measured with higher accuracy in a modal test [15]. An example containing the modeling of joints with uncertain parameters was shown in [16]. Li et al. $[17,18]$ used fuzzy theory and a genetic algorithm to identify joint parameters for modular robots. Friswell et al. [19] proposed a layer of special interface elements having material 


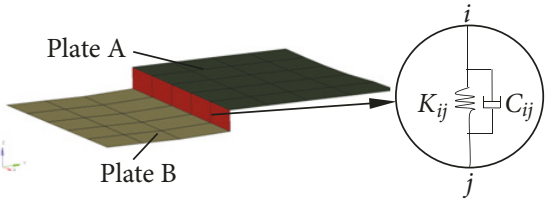

(a) Local seam-welding parameters description

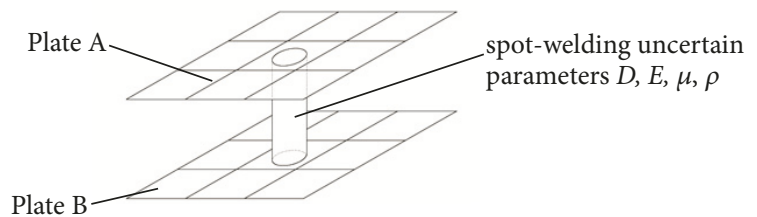

(b) Local spot-welding parameters description

FIGURE 1: Some example descriptions for joints parameters.

properties that may be adjusted to improve the prediction of the joint structures. Several parameters' selection methods were proposed in [20-22]; the high sensitive parameter was selected as the updating parameter, but a real uncertain or error parameter still needs to be further studied [7].

The rest of this article is organized as follows. Section 2 interprets the relation of joints parameters perturbation theory and dynamics, which reveals joints parameters selecting reason, illustrates some example descriptions for joints parameters, and introduces multiobjective optimization algorithms in identifying joints parameters. Sections 3 and 4 take seam-welding in an SUV frame and spot-welding in a thinwalled part of BIW as examples, respectively, to conduct structural actual dynamic tests and make some discussions. And in Section 5 summaries are drawn.

\section{Theory}

2.1. Some Example Descriptions for Joints Parameters. The first given description is seam-welding parameters in numerical modeling of an automotive frame. The uncertainty is embodied in parameters of thin interface elements. Young's modulus $E$, Poisson's ratio $\mu$, density $\rho$, and thickness $t$ of thin interface elements are regarded as uncertainty, which represent local rigidity $K_{i j}$. The uncertainty damping $C_{i j}$ of thin interface elements represents local damping. A segment schematic diagram of seam-welding is illustrated in Figure 1(a). The whole seam-welding structure damping is set to $G$, which is also unknown parameters in numerical modeling.

Another example description is spot-welding parameters as uncertainty in numerical modeling of thin-walled BIW parts. The uncertainty parameters are diameter $D$, Young's modulus $E$, Poisson's ratio $\mu$, density $\rho$, structural whole damping $G$, and local damping $C$ in connection positions. These parameters, as design variables, are determined by multiobjective optimization algorithm, which are determined by experience or simulating key parts of similar models in conventional modeling. Figure 1(b) shows a schematic diagram of spot-welding with joints parameters uncertainty.

2.2. Joints Parameters Perturbation Theory and Dynamics. In a free vibration system with multiple degrees of freedom, assume that some order circle frequency of system in numerical modeling is $\omega_{s}$ and vibration mode is $\left\{\varphi_{s}\right\}$, which obtained system dynamic experiment corresponding $\omega_{s}$ as $\omega_{t}$ and its vibration mode as $\left\{\varphi_{t}\right\}$. If simulation dynamic characteristics are needed to express actual ones, the simulation value $\omega_{s}$ must be updated to $\omega_{t}$ and corresponding vibration mode is changed from $\left\{\varphi_{s}\right\}$ to $\left\{\varphi_{t}\right\}$. Local corresponding rigidity, damping, and mass matrices are only needed to adjust $\Delta[K],[C]$, and $\Delta[M]$ to achieve that simulation behaviors describe actual ones in a local joints parameters identification problem. Actual stiffness matrix $[K]_{t}$ and actual mass matrix $[M]_{t}$ can be described as follows:

$$
\begin{aligned}
{[K]_{t} } & =[K]_{s}+\Delta[K], \\
{[M]_{t} } & =[M]_{s}+\Delta[M], \\
{[C]_{t} } & =[0]_{s}+[C],
\end{aligned}
$$

where $[K]_{S},[M]_{S}$, and $[0]_{S}$ represent simulation rigidity matrix, mass matrix, and damping matrix in conventional rigid numerical modeling, respectively.

The nature properties of matrices are used. Only local variable quantities $\Delta[K], \Delta[M]$, and $[C]$ are needed to be solved in (1) (3), and the whole system coefficient matrices are not necessary to be solved. The identification simulation model which can express structural actual dynamic characteristics can be described as follows:

$$
\begin{aligned}
& {\left[\left([K]_{s}+\Delta[K]\right)+j \omega_{t}[C]-\omega_{t}^{2}\left([M]_{s}+\Delta[M]\right)\right]\left\{\varphi_{t}\right\}} \\
& \quad=\{0\} .
\end{aligned}
$$

In order to analyze easier, frequency characteristic functions can be defined as

$$
\left[\alpha\left(\omega_{t}\right)\right]=\left([K]_{s}-\omega_{t}^{2}[M]_{s}\right)^{-1} .
$$

Substituting (5) to (4),

$$
\left\{\varphi_{t}\right\}=\left[\alpha\left(\omega_{t}\right)\right]\left(\omega_{t}^{2} \Delta[M]-\Delta[K]-j \omega_{t}[C]\right)\left\{\varphi_{t}\right\}
$$

If the mass of element between system nodes $i$ and $j$ needs to be updated by $\delta m_{i j}$, then, $\Delta[M]=\delta m_{i j}\left\{v_{m}^{(i j)}\right\}\left\{v_{m}^{(i j)}\right\}^{T}$, where $\left\{v_{m}^{(i j)}\right\}=(0,0, \ldots, 1, \ldots, 0)^{T}$. Similarly, if the joint stiffness of element between system nodes $i$ and $j$ needs to be updated by $\delta k_{i j}$, then, $\Delta[K]=\delta k_{i j}\left\{v_{n}^{(i j)}\right\}\left\{v_{n}^{(i j)}\right\}^{T}$, where $\left\{v_{n}^{(i j)}\right\}=$ $(0,0, \ldots, 1, \ldots, 0)^{T}$. If damping between system nodes $i$ and $j$ 
needs to be considered, $[C]=C_{i j}\left\{v_{l}^{(i j)}\right\}\left\{v_{l}^{(i j)}\right\}^{T}$ where $\left\{v_{l}^{(i j)}\right\}=$ $(0,0, \ldots, 1, \ldots, 0)^{T}$.

Assume that the total numbers of identification mass, rigidity, and damping elements are $m, n$, and $l$ in system, separately. Equation (6) can be transformed to the following:

$$
\begin{aligned}
& \left\{\varphi_{t}\right\}=\left[\alpha\left(\omega_{t}\right)\right]\left(\omega_{t}^{2} \sum_{i=1}^{m} \delta m_{i j}\left\{v_{m}^{(i j)}\right\}\left\{v_{m}^{(i j)}\right\}^{T}\right. \\
& \left.-\sum_{i=1}^{n} \delta k_{i j}\left\{v_{n}^{(i j)}\right\}\left\{v_{n}^{(i j)}\right\}^{T}-j \omega_{t} \sum_{i=1}^{l} C_{i j}\left\{v_{k}^{(i j)}\right\}\left\{v_{k}^{i j}\right\}^{T}\right) \\
& \cdot\left\{\varphi_{t}\right\}=\left[\alpha\left(\omega_{t}\right)\right]\left(\omega_{t}^{2} \sum_{i=1}^{m} \delta m_{i j}\left\{v_{m}^{(i j)}\right\}\left\{v_{m}^{(i j)}\right\}^{T}\right)\left\{\varphi_{t}\right\} \\
& X_{12}^{*}=X_{1}^{*}-X_{2}^{*}, X_{34}^{*}=X_{3}^{*}-X_{4}^{*}, \ldots, X_{i j}^{*}=X_{i}^{*}-X_{j}^{*}, \ldots, X_{2 n-12 n}^{*}=X_{2 n-1}^{*}-X_{2 n}^{*} \\
& \alpha_{p q, r s}\left(\omega_{t}\right)=\alpha_{p r}\left(\omega_{t}\right)+\alpha_{q s}\left(\omega_{t}\right)-\alpha_{p s}\left(\omega_{t}\right)-\alpha_{q r}\left(\omega_{t}\right) .
\end{aligned}
$$

$$
\begin{aligned}
& -\left[\alpha\left(\omega_{t}\right)\right]\left(\sum_{i=1}^{n} \delta k_{i j}\left\{v_{n}^{(i j)}\right\}\left\{v_{n}^{(i j)}\right\}^{T}\right)\left\{\varphi_{t}\right\}-\left[\alpha\left(\omega_{t}\right)\right] \\
& \cdot j \omega_{t}\left(\sum_{i=1}^{l} C_{i j}\left\{v_{k}^{(i j)}\right\}\left\{v_{k}^{(i j)}\right\}^{T}\right)\left\{\varphi_{t}\right\} .
\end{aligned}
$$

(1) For the situation that $n$ joints rigidity are updated only, the parts of identification rigidity elements in vibration modes $\left\{\varphi_{t}\right\}$ corresponding to certain order circle frequency $\omega_{t}$ can be described as $\left\{X_{12}^{*}, X_{34}^{*}, \ldots, X_{i j}^{*}, \ldots, X_{2 n-12 n}^{*}\right\}^{T}$, total of $n$ variables. The nodes matrix is converted to elements matrix. According to [23], the concrete forms of vibration mode transformation vectors $\left\{\varphi_{t}\right\}$ and frequency characteristic transformation functions $\alpha_{p q, r s}\left(\omega_{t}\right)$ are as follows:

Frequencies characteristic functions $\left[\alpha\left(\omega_{t}\right)\right]$ can be concrete described by the form of elements:

$$
\left[\begin{array}{cccccc}
\alpha_{12,12}\left(\omega_{t}\right) & \alpha_{12,34}\left(\omega_{t}\right) & \cdots & \alpha_{12, i j}\left(\omega_{t}\right) & \cdots & \alpha_{12,2 n-12 n}\left(\omega_{t}\right) \\
\alpha_{34,12}\left(\omega_{t}\right) & \alpha_{34,34}\left(\omega_{t}\right) & \cdots & \alpha_{34, i j}\left(\omega_{t}\right) & \cdots & \alpha_{34,2 n-12 n}\left(\omega_{t}\right) \\
\vdots & \vdots & \vdots & \vdots & \vdots & \vdots \\
\alpha_{i j, 12}\left(\omega_{t}\right) & \alpha_{i j, 34}\left(\omega_{t}\right) & \cdots & \alpha_{i j, i j}\left(\omega_{t}\right) & \cdots & \alpha_{i j, 2 n-12 n}\left(\omega_{t}\right) \\
\vdots & \vdots & \vdots & \vdots & \vdots & \vdots \\
\alpha_{2 n-12 n, 12}\left(\omega_{t}\right) & \alpha_{2 n-12 n, 34}\left(\omega_{t}\right) & \cdots & \alpha_{2 n-12 n, i j}\left(\omega_{t}\right) & \cdots & \alpha_{2 n-12 n, 2 n-12 n}\left(\omega_{t}\right)
\end{array}\right] .
$$

Equation (7) can be described as follows through a series of changes:

$$
\begin{aligned}
& \left\{X_{12}^{*}, X_{34}^{*}, \ldots, X_{i j}^{*}, \ldots, X_{2 n-12 n}^{*}\right\}^{T}=-\left[\begin{array}{cccccc}
\alpha_{12,12}\left(\omega_{t}\right) & \alpha_{12,34}\left(\omega_{t}\right) & \cdots & \alpha_{12, i j}\left(\omega_{t}\right) & \cdots & \alpha_{12,2 n-12 n}\left(\omega_{t}\right) \\
\alpha_{34,12}\left(\omega_{t}\right) & \alpha_{34,34}\left(\omega_{t}\right) & \cdots & \alpha_{34, i j}\left(\omega_{t}\right) & \cdots & \alpha_{34,2 n-12 n}\left(\omega_{t}\right) \\
\vdots & \vdots & \vdots & \vdots & \vdots & \vdots \\
\alpha_{i j, 12}\left(\omega_{t}\right) & \alpha_{i j, 34}\left(\omega_{t}\right) & \cdots & \alpha_{i j, i j}\left(\omega_{t}\right) & \cdots & \alpha_{i j, 2 n-12 n}\left(\omega_{t}\right) \\
\vdots & \vdots & \vdots & \vdots & \vdots & \vdots \\
\alpha_{2 n-12 n, 12}\left(\omega_{t}\right) & \alpha_{2 n-12 n, 34}\left(\omega_{t}\right) & \cdots & \alpha_{2 n-12 n, i j}\left(\omega_{t}\right) & \cdots & \alpha_{2 n-12 n, 2 n-12 n}\left(\omega_{t}\right)
\end{array}\right] \\
& \times\left[\begin{array}{ccccc}
\delta k_{12} & & & & \\
& \delta k_{34} & & & \\
& & \ddots & & \\
& & \delta k_{i j} & & \\
& & & \ddots & \\
& & & & \delta k_{2 n-12 n}
\end{array}\right]\left\{X_{12}^{*}, X_{34}^{*}, \ldots, X_{i j}^{*}, \ldots, X_{2 n-12 n}^{*}\right\}^{T} .
\end{aligned}
$$


Its general solution is

$$
\begin{gathered}
-\alpha_{i j, 12}\left(\omega_{t}\right) \delta k_{12} X_{12}^{*}-\alpha_{i j, 34}\left(\omega_{t}\right) \delta k_{34} X_{34}^{*}-\cdots \\
-\alpha_{i j, i j}\left(\omega_{t}\right) \delta k_{i j} X_{i j}^{*}-\cdots \\
-\alpha_{i j, 2 n-12 n}\left(\omega_{t}\right) \delta k_{2 n-12 n} X_{2 n-12 n}^{*}=X_{i j}^{*} .
\end{gathered}
$$

When rigidity between $i$ and $j$ is updated only, then $\delta k_{12}=0, \ldots, \delta k_{i-1 j-1}=0, \delta k_{i+1 j+1}=0, \ldots, \delta k_{2 n-12 n}=0$. Equation (11) can be converted to

$$
\delta k_{i j}=-\frac{1}{\alpha_{i j, i j}\left(\omega_{t}\right)} .
$$

(2) Similarly, the general solution of local mass matrices with $m$ elements identification can be derived as follows:

$$
\begin{aligned}
\omega_{t}^{2} & {\left[\alpha_{i j, 12}\left(\omega_{t}\right) \delta m_{12} X_{12}^{*}+\alpha_{i j, 34}\left(\omega_{t}\right) \delta m_{34} X_{34}^{*}+\cdots\right.} \\
& +\alpha_{i j, i j}\left(\omega_{t}\right) \delta m_{i j} X_{i j}^{*}+\cdots \\
& \left.+\alpha_{i j, 2 m-12 m}\left(\omega_{t}\right) \delta m_{2 m-12 m} X_{2 m-12 m}^{*}\right]=X_{i j}^{*}
\end{aligned}
$$

When element mass between $i$ and $j$ is updated only, then

$$
\delta m_{i j}=\frac{1}{\alpha_{i j, i j}\left(\omega_{t}\right)} \cdot \frac{1}{\omega_{t}^{2}} .
$$

(3) Similarly, the general solution of local damping matrices with $l$ elements identification can be derived as follows:

$$
\begin{aligned}
j \omega_{t} & {\left[-\alpha_{i j, 12}\left(\omega_{t}\right) C_{12} X_{12}^{*}-\alpha_{i j, 34}\left(\omega_{t}\right) C_{34} X_{34}^{*}-\cdots\right.} \\
& -\alpha_{i j, i j}\left(\omega_{t}\right) C_{i j} X_{i j}^{*}-\cdots \\
& \left.-\alpha_{i j, 2 n-12 n}\left(\omega_{t}\right) C_{2 n-12 n} X_{2 n-12 n}^{*}\right]=X_{i j}^{*} .
\end{aligned}
$$
then

When element damping between $i$ and $j$ is updated only,

$$
C_{i j}=-\frac{1}{\alpha_{i j, i j}\left(\omega_{t}\right)} \cdot \frac{1}{j \omega_{t}} .
$$

The updated quantities of rigidity, mass, or damping through such pair of $i$ - $j$ element can be solved by (12), (14), or (16). The updated quantities of rigidity matrices, mass matrices, or damping matrices through some pairs of local elements can be solved by general solution (11), (13), or (15). This is the theoretical interpretation for property parameters selecting for identification.

\subsection{Multiobjective Optimization Algorithms in Joint Param-} eters Identification. Multiobjective optimization algorithms are utilized to update the uncertain joints parameters. What is critical is that the parameters are assumed uncertain and the identification process is approached actual conditions by various multiobjective optimization algorithms. In the seam-welding example in present research, Nondominated Sorting Genetic Algorithm (NSGA-II) as one of the most ideal multiobjective evolutionary algorithms [24] is used in automotive frame dynamics with joints parameters uncertain and Adaptive Multipopulation Genetic Algorithm (AMGA) in the spot-welding example of automotive thin-walled BIW parts dynamics with parameters uncertain is used.

The optimization problems of identification in the illustrated samples can be expressed by mathematical model (17) and (18) separately:

$$
\begin{array}{ll}
\text { target } & \omega_{f s i}=\omega_{f t i} \quad(i=1,2, \ldots, 6) \\
\text { var. } & E^{L} \leq E \leq E^{U} \\
& t^{L} \leq t \leq t^{U} \\
& \mu^{L} \leq \mu \leq \mu^{U} \\
& \rho^{L} \leq \rho \leq \rho^{U} \\
& G^{L} \leq G \leq G^{U} \\
& C^{L} \leq C \leq C^{U},
\end{array}
$$

where $\omega_{f s i}, \omega_{f t i}$ are the $i$ th simulation and test frequencies of automotive frame separately. $E, t, \mu, \rho$ are Young's modulus, thickness, Poisson's ratio, and density of local seam-welding. $G$ and $C$ represent damping of the whole structure and local seam-welding. The high and low corner mark represent the lower and upper bounds separately:

$$
\begin{array}{ll}
\text { target } & \omega_{p s i}=\omega_{p t i} \quad(i=1,2,3) \\
\text { var. } & E^{L} \leq E \leq E^{U} \\
& D^{L} \leq D \leq D^{U} \\
& \mu^{L} \leq \mu \leq \mu^{U} \\
& \rho^{L} \leq \rho \leq \rho^{U} \\
& G^{L} \leq G \leq G^{U},
\end{array}
$$

where $\omega_{p s i}, \omega_{p t i}$ are the $i$ th simulation and test frequencies of automotive thin-walled BIW parts, separately. $D, E, \mu$, $\rho$, and $G$ represent spot-welding diameter, Young's modulus, Poisson's ratio, density, and damping in this spot-welding example.

\section{Seam-Welding in an SUV Frame}

Experimental modal is conducted to an SUV frame to investigate the structural actual dynamics. The test is carried out in a whole automotive production and test workshop of a company in China. Nondamping elastic rope is used to suspend the frame to free state and a schematic diagram is illustrated in Figure 2(a). The method of single-point exciting and multipoint collecting signals is used. 60 test points in the area of structural key connections or response positions but not modal order nodes are taken as measurement response points. Some test points are illustrated in Figure 2(b). The signals of exciting force and acceleration are transported to 


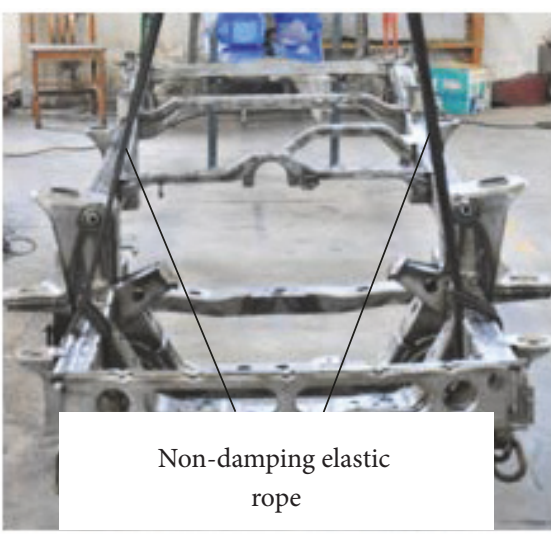

(a) Suspend mode

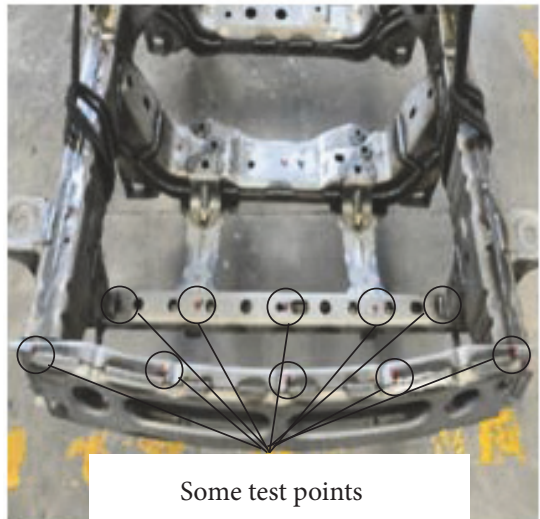

(b) Test points

FIGURE 2: An SUV frame experiment.

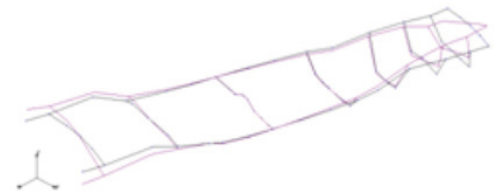

(a) 1st mode

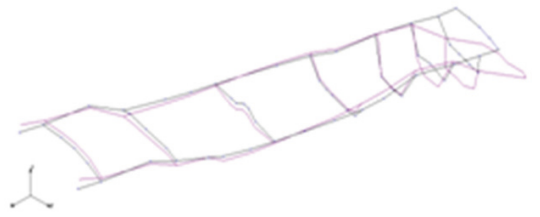

(d) 4th mode

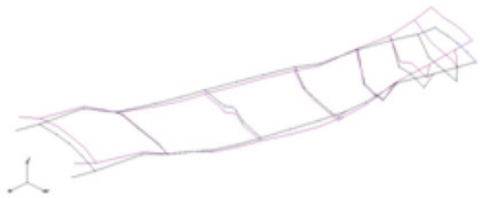

(b) 2nd mode

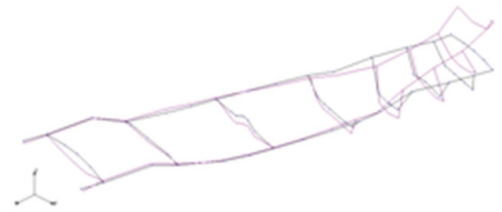

(e) 5th mode

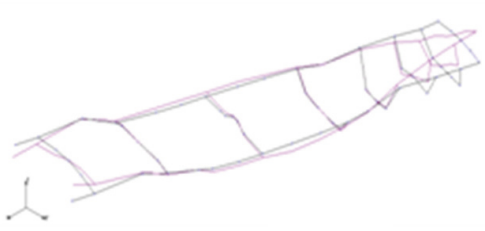

(c) 3rd mode

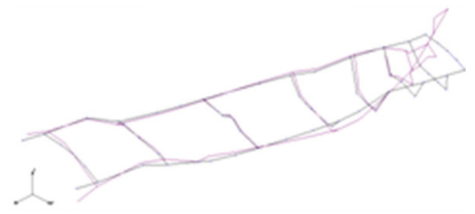

(f) 6th mode

Figure 3: Experimental vibration modes $\left\{\varphi_{t}\right\}$.

computer-operated acquisition system through power amplifier and the experimental modal parameters are recognized by fitting gathered data. The actual modal nature frequencies $\omega_{f t i}$ and vibration modes $\left\{\varphi_{t}\right\}$ are obtained as optimization targets for numerical modeling with joint parameters identification.

All orders modal frequencies $\omega_{f t i}$ and vibration modes $\left\{\varphi_{t}\right\}$ of the SUV frame are acquired by dealing with experimental data. The lowest six-order modal dates and vibration modes are illustrated as follows: the first order is the first torsion, $22.46 \mathrm{~Hz}$; the second order is the first bending, $29.30 \mathrm{~Hz}$; the third order is the second torsion, $44.92 \mathrm{~Hz}$; the fourth order is the second bending, $66.41 \mathrm{~Hz}$; the fifth order is bending-torsion complex, $71.29 \mathrm{~Hz}$; and the sixth order is the third torsion, $82.03 \mathrm{~Hz}$, respectively. The vibration modes are illustrated in Figure 3.

The MAC value of each order frequency is shown in Figure 4, frequency order is in horizontal and vertical coordinates, and the MAC value is in upright coordinates of Cartesian coordinate system.

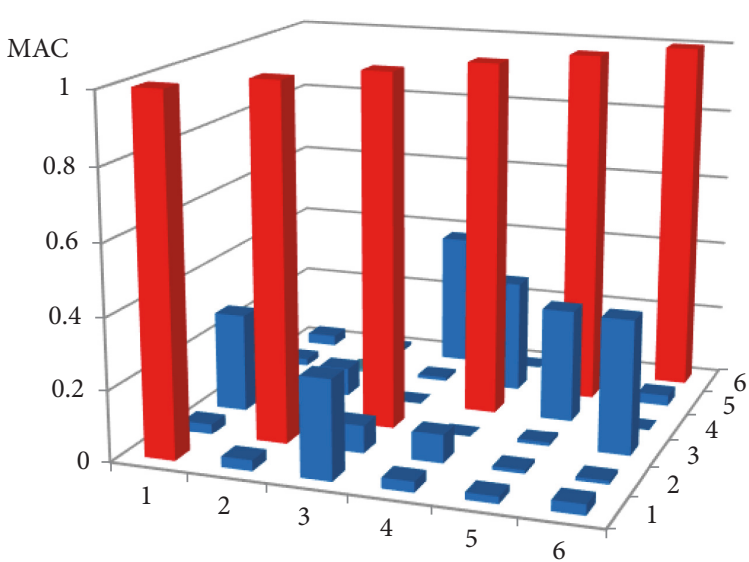

Figure 4: MAC of test modal.

Young's modulus, thickness, Poisson's ratio, density, damping of local seam-welding thin plate, and damping of the 

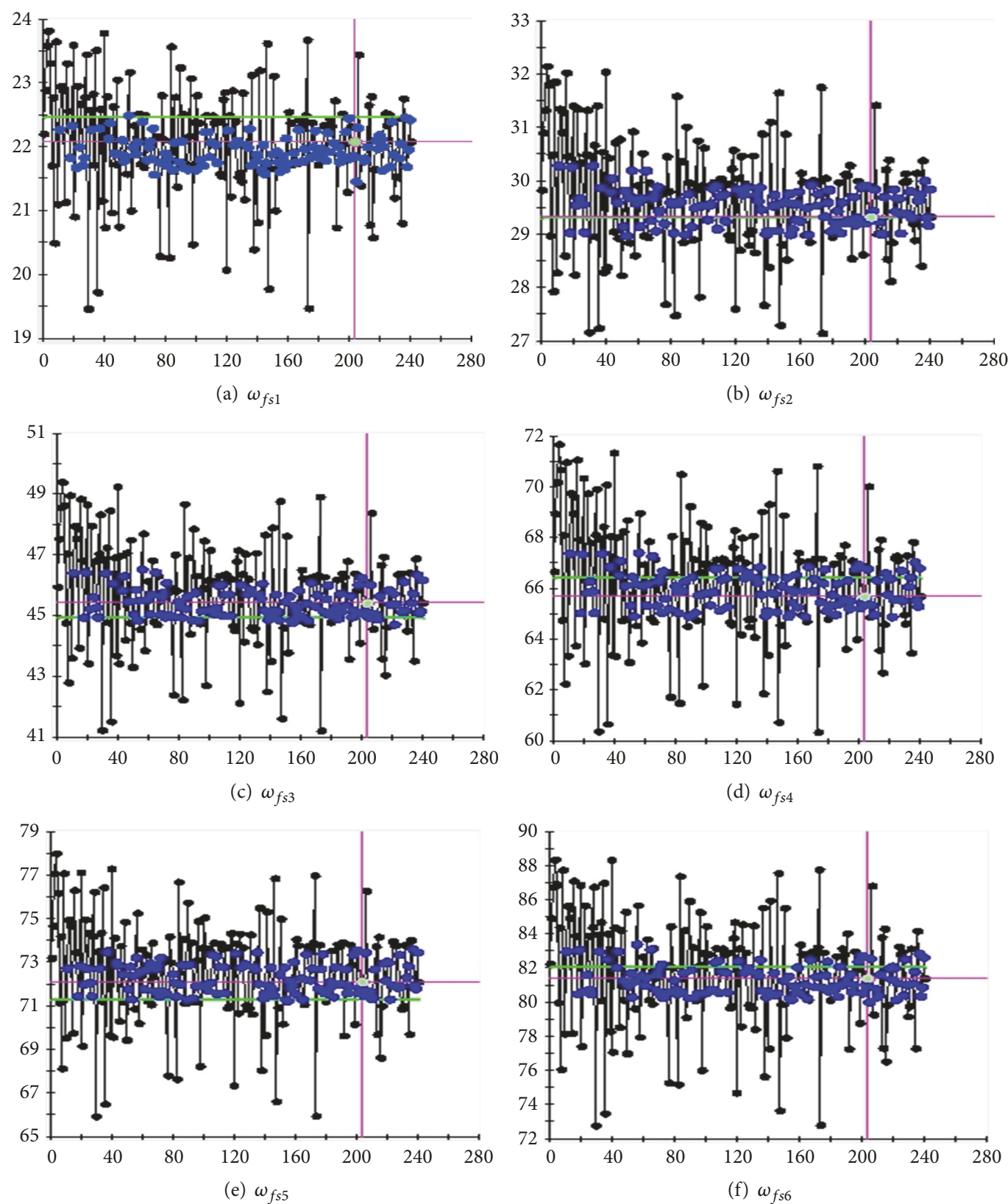

FIGURE 5: The iteration processes of objectives.

whole structure are variable; the lowest six-order frequencies of experimental value $\omega_{f t i}$ are objectives. NSGA-II is used to solve this inverse parameters problem. 242 iterations are conducted. The iteration processes of objectives are shown in Figure 5. The improvement Pareto results are illustrated in Table 1 and the 204th step is the most suitable parameters solution for dynamics simulation of the example structural seam-welding. Horizontal coordinates is iterative steps, and vertical coordinates is frequency. Unit of vertical coordinates is $\mathrm{Hz}$.

Finite elements analysis solver MSC.Nastran is used for validation. The lowest six-order nonrigid frequencies are selected to investigate the differences of dynamics between two numerical methodologies. The frequencies $\omega_{f s i}$ and vibration modes $\left\{\varphi_{s}\right\}$ are obtained. Results of dynamics with rigid joints are illustrated as follows: the first order is the first torsion, $21.32 \mathrm{~Hz}$; the second order is the first bending, $28.66 \mathrm{~Hz}$; the third order is the second torsion, $44.25 \mathrm{~Hz}$; the fourth order is the second bending, $63.46 \mathrm{~Hz}$; the fifth order is bending-torsion complex, $70.47 \mathrm{~Hz}$; and the sixth order is the third torsion, $79.40 \mathrm{~Hz}$, respectively. The vibration modes are illustrated in Figure 6.

The frequencies and vibration modes generated by multiobjective identification are obtained under the same situation as rigid one, whose results are as follows: the first order is the first torsion, $22.25 \mathrm{~Hz}$; the second order is the first bending, 
TABle 1: The improvement Pareto results.

\begin{tabular}{|c|c|c|c|c|c|c|}
\hline Runs & $E / G P a$ & $t / \mathrm{mm}$ & $\mu$ & $\rho / \mathrm{g} / \mathrm{cm}^{3}$ & C & $G$ \\
\hline 1 & 36.13 & 4.59 & 0.42 & 6.10 & 0.44 & 0.68 \\
\hline 18 & 370.67 & 0.77 & 0.49 & 6.65 & 0.63 & 0.35 \\
\hline 22 & 36.13 & 4.17 & 0.20 & 6.10 & 0.44 & 0.68 \\
\hline 24 & 36.13 & 4.59 & 0.42 & 6.93 & 0.44 & 0.68 \\
\hline 59 & 36.13 & 5.15 & 0.42 & 6.93 & 0.44 & 0.62 \\
\hline 186 & 69.87 & 5.67 & 0.14 & 5.14 & 0.54 & 0.43 \\
\hline 190 & 471.21 & 0.77 & 0.49 & 6.65 & 0.63 & 0.35 \\
\hline 204 & 370.59 & 1.27 & 0.49 & 5.61 & 0.69 & 0.35 \\
\hline
\end{tabular}

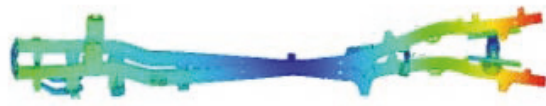

(a) 1st mode

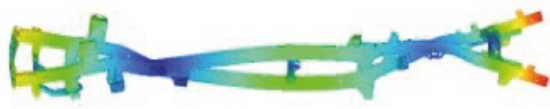

(c) 3rd mode

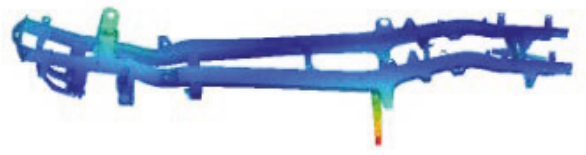

(e) 5th mode

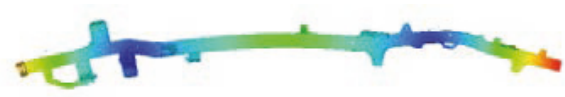

(b) 2nd mode

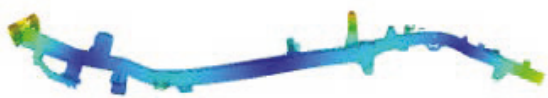

(d) 4th mode

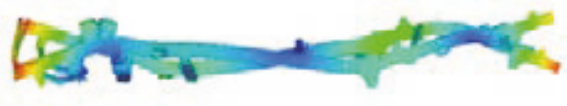

(f) 6th mode

FIGURE 6: Vibration modes of rigid modeling methodology.

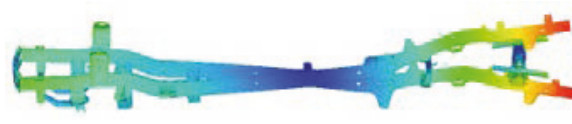

(a) 1st mode

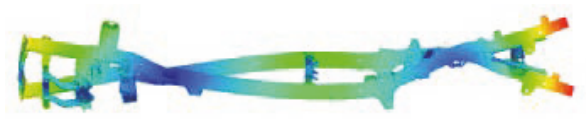

(c) 3rd mode

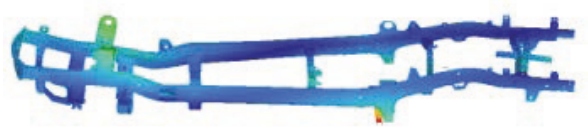

(e) 5th mode

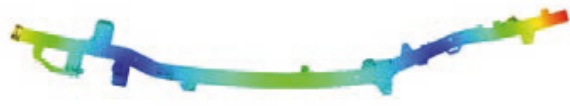

(b) 2nd mode

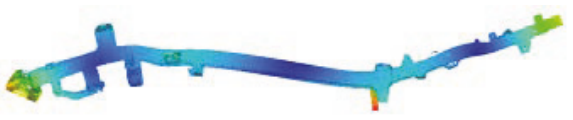

(d) 4th mode

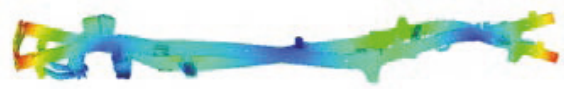

(f) 6th mode

FIgURE 7: Vibration modes of MOD modeling methodology.

$29.72 \mathrm{~Hz}$; the third order is the second torsion, $45.41 \mathrm{~Hz}$; the fourth order is the second bending, $66.40 \mathrm{~Hz}$; the fifth order is bending-torsion complex, $72.10 \mathrm{~Hz}$; and the sixth order is the third torsion, $82.08 \mathrm{~Hz}$, respectively. The vibration modes are illustrated in Figure 7. Multiobjective optimization algorithm modeling methodology is abbreviated to MOD.

From the above numerical results we can see that there are differences deviations of $0.93 \sim 2.94 \mathrm{~Hz}$ for the lowest sixorder frequencies in numerical analyses of seam-welding simulations by rigid and MOD modeling methodologies. Vibration modes of the lowest six-order models are in full agreement: the first order is the first torsion, the second order is the first bending, the third order is the second torsion, the fourth order is the second bending, the fifth order is bendingtorsion complex, and the sixth order is the third torsion.

Figure 8 shows the comparison of the lowest six experimental modals and analysis modals obtained by rigid and MOD modeling methodologies as well as the trends 
TABLE 2: Comparison of concrete frequency error.

\begin{tabular}{lcccccr}
\hline Error/Hz & & \multicolumn{3}{c}{ Order } & 4th & 5th \\
\hline Conventional & 1st & 2nd & 0.64 & 0.67 & 2.95 & 0.82 \\
MOD & 1.14 & 0.42 & 0.49 & 0.01 & 0.91 & 0.05 \\
\hline
\end{tabular}

TABLE 3: Improvement Pareto solutions.

\begin{tabular}{|c|c|c|c|c|c|}
\hline Runs & $D / \mathrm{mm}$ & $E / \mathrm{GPa}$ & $\mu$ & $\rho /\left(\mathrm{g} / \mathrm{cm}^{3}\right)$ & $G$ \\
\hline 1 & 3.5 & 619.0 & 0.41 & 2.7 & 0.98 \\
\hline 4 & 9.5 & 133.5 & 0.18 & 1.9 & 0.35 \\
\hline 5 & 2.1 & 569.0 & 0.33 & 1.6 & 0.28 \\
\hline 9 & 5.5 & 45.0 & 0.18 & 5.7 & 0.20 \\
\hline 11 & 2.0 & 974.0 & 0.25 & 2.3 & 0.13 \\
\hline 21 & 1.5 & 831.0 & 0.16 & 7.8 & 0.044 \\
\hline 31 & 1.2 & 387.0 & 0.08 & 4.1 & 0.44 \\
\hline 91 & 1.1 & 828.0 & 0.37 & 8.2 & 0.089 \\
\hline 136 & 1.2 & 587.5 & 0.05 & 5.1 & 0.13 \\
\hline 240 & 1.1 & 831.0 & 0.02 & 4.1 & 0.12 \\
\hline
\end{tabular}

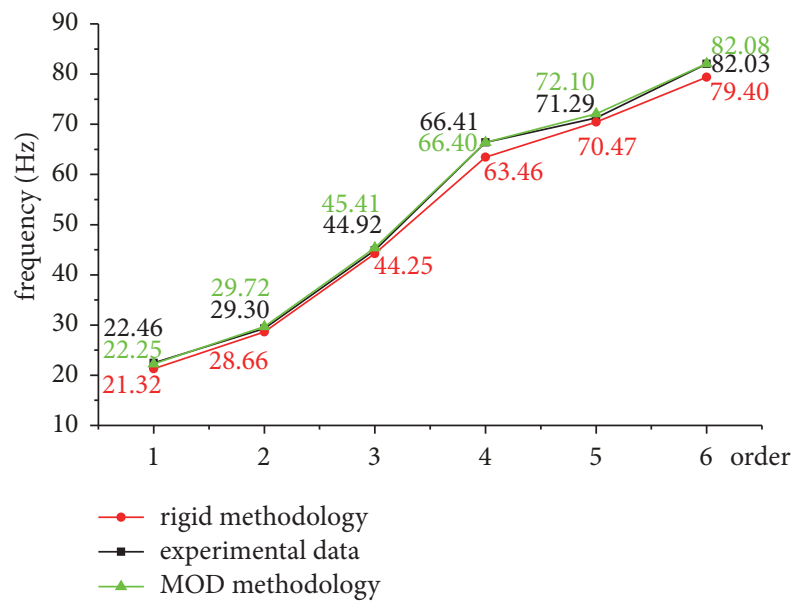

FIGURE 8: The overall comparison and trends graph.

graph. The overall comparison, concrete frequencies data, and trends are listed. All order frequencies obtained by MOD modeling methodology are more accurate than rigid modeling within experimental frequencies. The maximum frequency error in rigid modeling methodology is $2.95 \mathrm{~Hz}$ which appeared in the fourth order. The error value of the sixth order is $2.63 \mathrm{~Hz}$, also larger than other orders. The error value of the first order is $1.14 \mathrm{~Hz}$. However, in MOD modeling methodology, the error value of the fourth order is reduced to $0.01 \mathrm{~Hz}$, the largest error is $0.91 \mathrm{~Hz}$ which appeared in the fifth order, and the error value of the first order reduced to $0.21 \mathrm{~Hz}$. The concrete frequency error of the lowest six orders are shown in Table 2. The correctness and accuracy of MOD modeling methodology to simulate seam-welding are effectively verified.

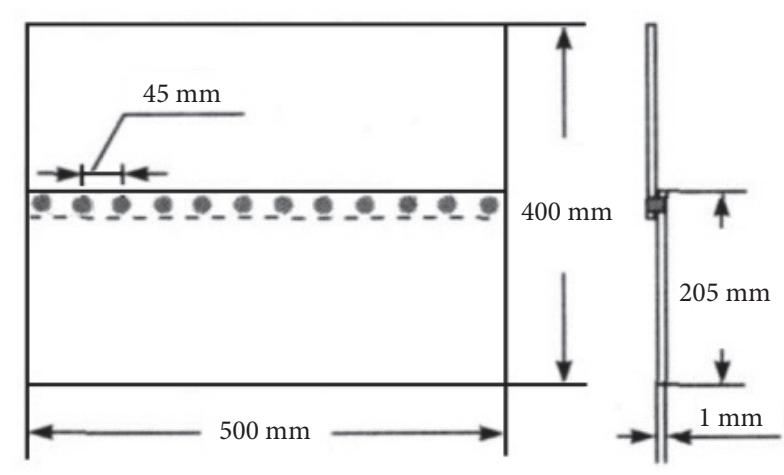

FIGURE 9: Geometric model for spot-welding plate.

\section{Spot-Welding in a Thin-Walled Part of BIW}

Thin-walled part of $1 \mathrm{~mm}$ with $45 \mathrm{~mm}$ space spot-welding and its modal experiment were studied by Gao et al. [25]. The lowest three-order modal frequencies and vibration modes are the first torsion, $16.64 \mathrm{~Hz}$; the longitudinal first bending, $26.42 \mathrm{~Hz}$; the horizontal first bending, $32.23 \mathrm{~Hz}$. Geometric model and experimental modal vibration modes are shown as Figures 9 and 10.

Diameter $D$, Young's modulus $E$, Poisson's ratio $\mu$, density $\rho$ of spot-welding parameters, and structural damping $G$ are selected as variables for identification according to the reason in Section 2. The lowest three-order actual frequencies are objectives. AMGA embedded in Isight software is used to approach actual conditions. 501 iterations are carried out. The iteration processes of objectives are shown in Figure 11. Horizontal coordinates are iterative steps, and vertical coordinates are frequency. Unit of vertical coordinates is $\mathrm{Hz}$. The improvement Pareto results are illustrated in Table 3 and 


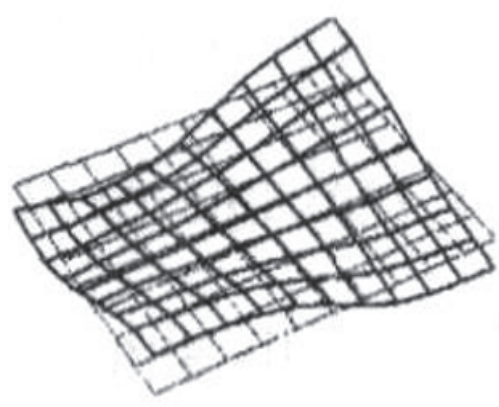

(a) Torsion

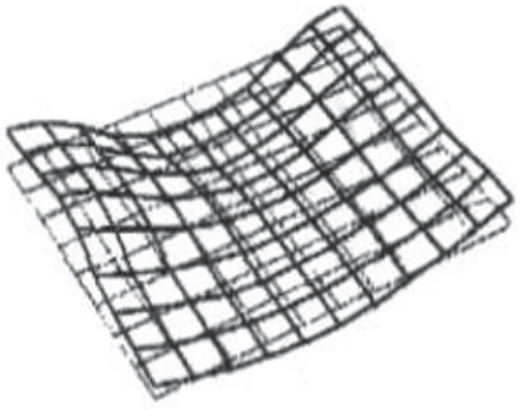

(b) Longitudinal bending

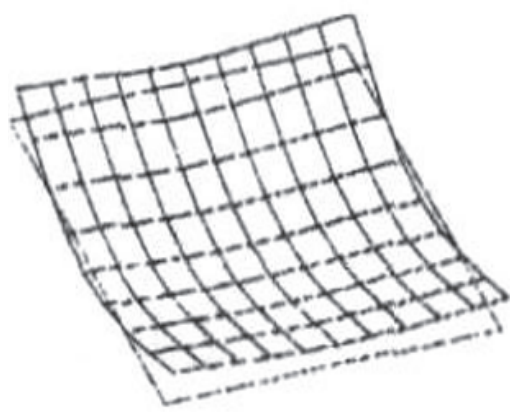

(c) Horizontal bending

FIGURE 10: Vibration modes for experimental modal.

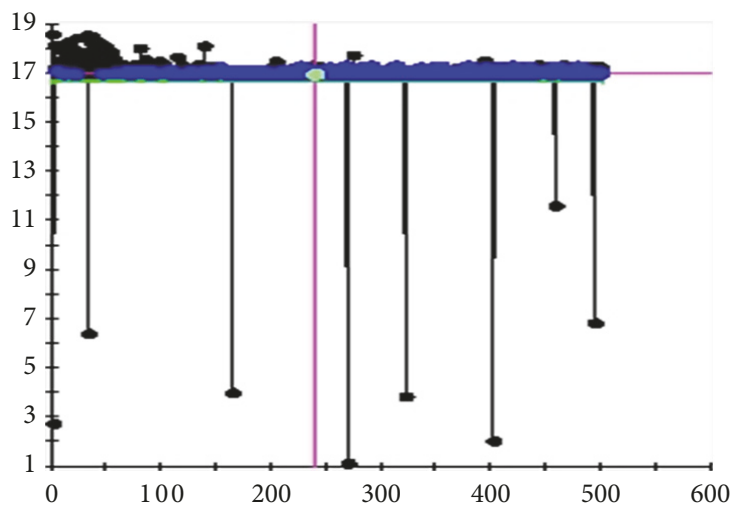

(a) $\omega_{p s 1}$

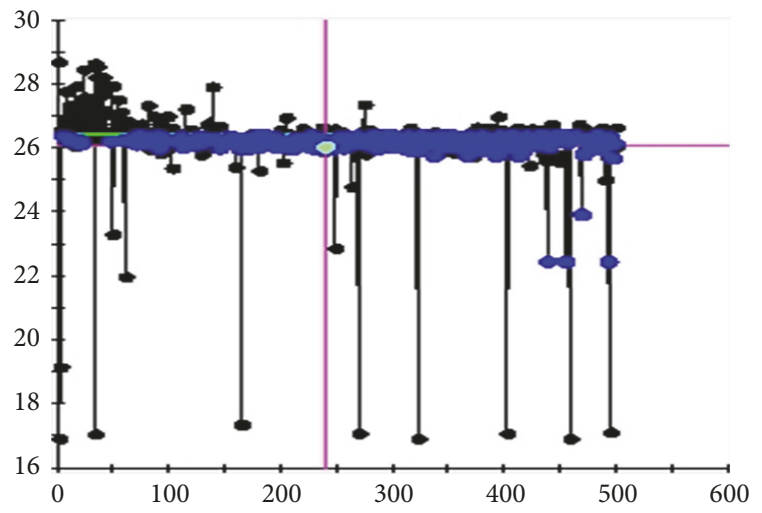

(b) $\omega_{p s 2}$

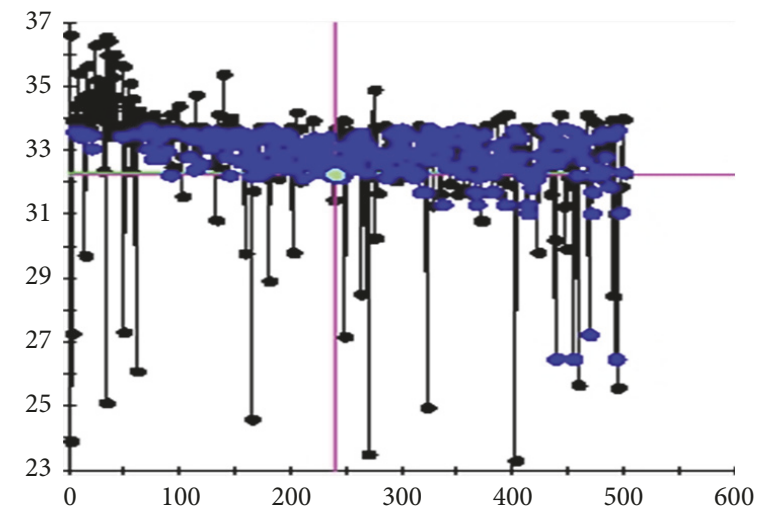

(c) $\omega_{p s 3}$

FIGURE 11: Iterative processes of objectives.

the 240th step, which is the 10th in improvement Pareto results, is the more suitable parameters approaching solution in MOD modeling methodology for dynamics of the example structural with spot-welding. So, rigidity variable is calibrated through Young's modulus $E$, diameter $D$, density $\rho$, and Poisson's ratio $\mu$. Damping variables are calibrated through structural damping $G$.

Conventional modeling methodology and MOD modeling methodology are used to establish finite element models and separately to analyze modal. Eigenvalues and vibration modes of $1 \mathrm{~mm}$ steel plate with spot-welding are obtained.
The lowest 3-order frequencies and vibration modes of two modeling methodology are illustrated in Figures 12 and 13. The concrete comparison of two methodologies are in Table 4.

The frequencies of two methodologies are both in agreement with test values. The lowest 3-order vibration modes of MOD modeling methodology are more consistent with test modes than conventional modeling. The first and third vibration modes with rigid modeling are different by 180 degrees with test vibration modes. Complex modal considering structural damping is more accurate in solving engineering problems. 
TABLE 4: Comparisons of two spot-welding modeling methodologies.

\begin{tabular}{|c|c|c|c|}
\hline order & actual & rigid & MOD \\
\hline \multicolumn{4}{|l|}{1} \\
\hline Vibration mode & torsion & torsion & torsion \\
\hline frequency/Hz & 16.64 & 16.92 & 16.92 \\
\hline Error/Hz & - & 0.28 & 0.28 \\
\hline \multicolumn{4}{|l|}{2} \\
\hline Vibration mode & longitudinal bending & longitudinal bending & longitudinal bending \\
\hline frequency/Hz & 26.42 & 25.81 & 26.09 \\
\hline Error/Hz & - & -0.61 & -0.33 \\
\hline \multicolumn{4}{|l|}{3} \\
\hline Vibration mode & horizontal bending & horizontal bending & horizontal bending \\
\hline frequency/Hz & 32.23 & 31.84 & 32.31 \\
\hline Error $/ \mathrm{Hz}$ & - & -0.39 & 0.08 \\
\hline
\end{tabular}

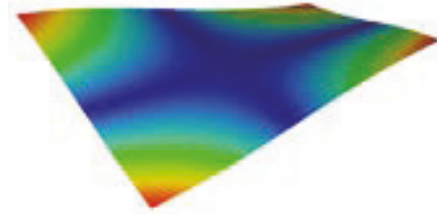

(a) 1 st mode $(16.92 \mathrm{~Hz})$

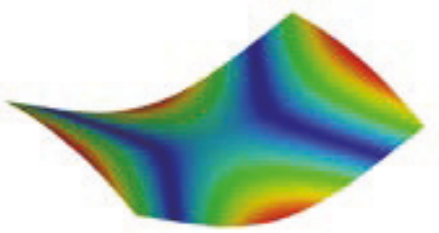

(b) 2nd mode $(25.81 \mathrm{~Hz})$

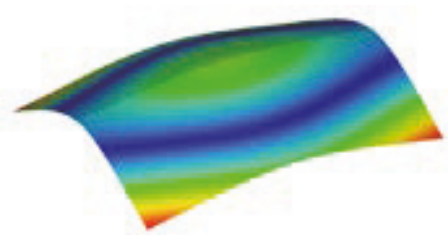

(c) 3rd mode $(31.84 \mathrm{~Hz})$

FIGURE 12: The lowest 3-order modal of conventional modeling.

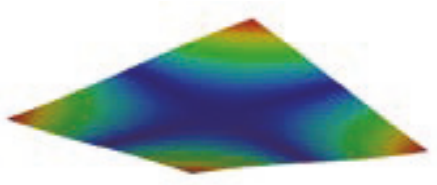

(a) 1 st mode $(16.92 \mathrm{~Hz})$

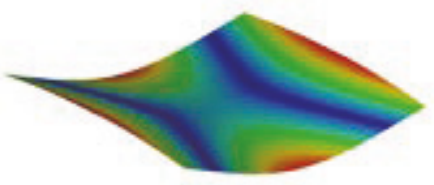

(b) 2nd mode $(26.09 \mathrm{~Hz})$

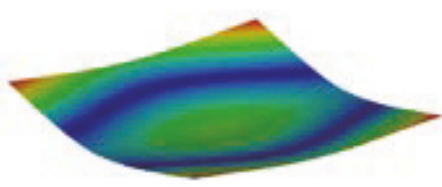

(c) 3rd mode $(32.31 \mathrm{~Hz})$

FIGURE 13: The lowest 3-order modal of MOD modeling.

\section{Conclusions}

(1) In present work, multiobjective optimization algorithms (MOD) are used to identify joints parameters by approaching actual test values in each series of structure to decrease unreliability or error for the numerical modeling, and it can also improve the simulation technical capability and achieve more hope of reference value.

(2) Taking automotive dynamics with seam-welding and spot-welding as examples, the relationship of joints parameters perturbation and structural dynamics is derived to give the selecting reason of parameters for identification.

(3) In application of local seam-welding within an SUV frame with MOD modeling, all the investigated order frequencies through MOD are more accurate than conventional rigid values. The maximum error is $0.91 \mathrm{~Hz}$ and the first order is $0.21 \mathrm{~Hz}$ in MOD modeling methodology while the maximum error is $2.95 \mathrm{~Hz}$ and the first order is $1.14 \mathrm{~Hz}$ in conventional rigid methodology.

(4) In application of local spot-welding within automotive thin-walled part in BIW, the first and third vibration modes with rigid modeling are of 180 degrees different with test vibration modes. The MOD modeling methodologies are more in agreement with test modes than conventional modeling.

\section{Data Availability}

All relevant data used to support the findings of this study are included within the article. The models used to support the findings of this study are available from the author, Yuedong Yang, upon request according to certain rules.

\section{Conflicts of Interest}

The authors declare that there are no conflicts of interest regarding the publication of this paper. 


\section{Acknowledgments}

This research is supported by the National Natural Science Foundation of China (51775193) and the Science and Technology Planning Project of Guangdong Province China (2015B010137002; 2016A050503021; 2016B090918089).

\section{References}

[1] M. Sanati, Y. Alammari, J. H. Ko, and S. S. Park, "Identification of joint dynamics in lap joints," Archive of Applied Mechanics, vol. 87, no. 1, pp. 99-113, 2017.

[2] M. J. Ratcliffe and N. A. J. Lieven, "A generic element-based method for joint identification," Mechanical Systems and Signal Processing, vol. 14, no. 1, pp. 3-28, 2000.

[3] J. C. Awkar and E. M. Lui, "Seismic analysis and response of multistory semirigid frames," Engineering Structures, vol. 21, no. 5, pp. 425-441, 1999.

[4] D. J. Ewins, Modal testing: theory and practice, Research Studies Press, Letchworth, England, 1984.

[5] V. Arora, "Comparative study of finite element model updating methods," Journal of Vibration and Control, vol. 17, no. 13, pp. 2023-2039, 2011.

[6] S. Bograd, P. Reuss, A. Schmidt, L. Gaul, and M. Mayer, "Modeling the dynamics of mechanical joints," Mechanical Systems and Signal Processing, vol. 25, no. 8, pp. 2801-2826, 2011.

[7] Z. Huang, C. Zang, Y. Jiang, and X. Wang, "Dynamic finite element model validation of an assembled aero-engine casing," Journal of Physics: Conference Series, vol. 744, IOP Publishing, no. 1, Article ID 012139, 2016.

[8] T. Yang, S.-H. Fan, and C.-S. Lin, "Joint stiffness identification using FRF measurements," Computers \& Structures, vol. 81, no. 28-29, pp. 2549-2556, 2003.

[9] D.-H. Lee and W.-S. Hwang, "An identification method for joint structural parameters using an FRF-based substructuring method and an optimization technique," Journal of Mechanical Science and Technology, vol. 21, no. 12, pp. 2011-2022, 2007.

[10] K.-T. Yang and Y.-S. Park, "Joint structural parameter identification using a subset of frequency response function measurements," Mechanical Systems and Signal Processing, vol. 7, no. 6, pp. 509-530, 1993.

[11] J.-S. Tsai and Y.-F. Chou, "The identification of dynamic characteristics of a single bolt joint," Journal of Sound and Vibration, vol. 125 , no. 3, pp. 487-502, 1988.

[12] F. Hu, B. Wu, Y. Hu, and T. Shi, "Identification of dynamic stiffness matrix of bearing joint region," Frontiers of Mechanical Engineering in China, vol. 4, no. 3, pp. 289-299, 2009.

[13] J. H. Wang and C. M. Liou, "Experimental identification of mechanical joint parameters," Journal of Vibration and Acoustics, vol. 113, no. 1, pp. 28-36, 1991.

[14] H. Y. Hwang, "Identification techniques of structure connection parameters using frequency response functions," Journal of Sound and Vibration, vol. 212, no. 3, pp. 469-479, 1998.

[15] W. L. Li, "A new method for structural model updating and joint stiffness identification," Mechanical Systems and Signal Processing, vol. 16, no. 1, pp. 155-167, 2002.

[16] M. Hanss, S. Oexl, and L. Gaul, "Identification of a boltedjoint model with fuzzy parameters loaded normal to the contact interface," Mechanics Research Communications, vol. 29, no. 2-3, pp. 177-187, 2002.
[17] Y. Li, X. Liu, Z. Peng, and Y. Liu, “The identification of joint parameters for modular robots using fuzzy theory and a genetic algorithm," Robotica, vol. 20, no. 5, pp. 509-517, 2002.

[18] Y. Li, X. Liu, Z. Peng, and Y. Liu, "Joint parameters identification for redundant manipulators based on fuzzy theory and genetic algorithm," in Canadian Conference on Electrical and Computer Engineering, 2002, IEEE CCECE 2002, vol. 1, pp. 560-565, Winnipeg, Manitoba, Canada, 2002.

[19] M. I. Friswell, J. E. Penny, and S. D. Garvey, "Parameter subset selection in damage location," Inverse Problems in Engineering, vol. 5, no. 3, pp. 189-215, 1997.

[20] H. Ahmadian, J. E. Mottershead, S. James, M. I. Friswell, and C. A. Reece, "Modelling and updating of large surface-to-surface joints in the AWE-MACE structure," Mechanical Systems and Signal Processing, vol. 20, no. 4, pp. 868-880, 2006.

[21] G.-H. Kim and Y.-S. Park, "An automated parameter selection procedure for finite-element model updating and its applications," Journal of Sound and Vibration, vol. 309, no. 3-5, pp. 778793, 2008.

[22] G.-H. Kim and Y.-S. Park, "An improved updating parameter selection method and finite element model update using multiobjective optimisation technique," Mechanical Systems and Signal Processing, vol. 18, no. 1, pp. 59-78, 2004.

[23] X. C. Zhang and G. Z. Sun, "A modal approximation method of frequency modification," in Proceedings of the Asia-Pacific Vibration Conference, vol. 93, pp. 402-408, 1993.

[24] K. Deb, A. Pratap, S. Agarwal, and T. Meyarivan, "A fast and elitist multiobjective genetic algorithm: NSGA-II," IEEE Transactions on Evolutionary Computation, vol. 6, no. 2, pp. 182197, 2002.

[25] S.-N. Gao, Z.-X. Deng, and Y.-M. Hu, "A study on the finite element modeling for spot-welds of vehicle body," Automotive Engineering, vol. 30, no. 9, pp. 811-815, 2008 (Chinese). 


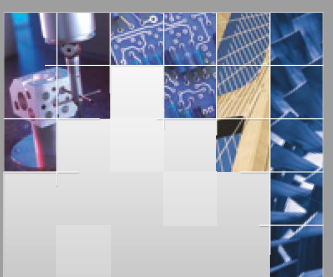

\section{Enfincering}
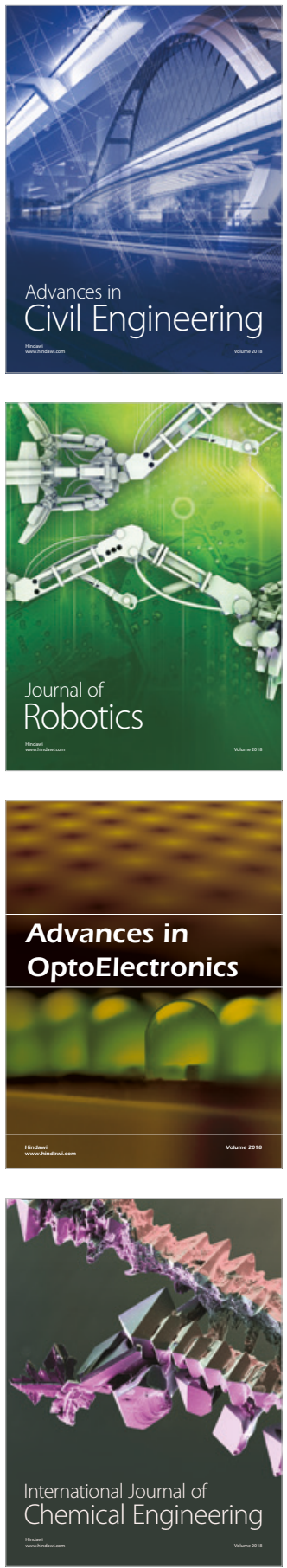

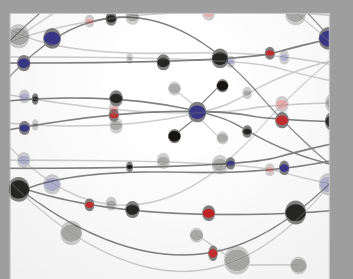

\section{Rotating \\ Machinery}

The Scientific World Journal

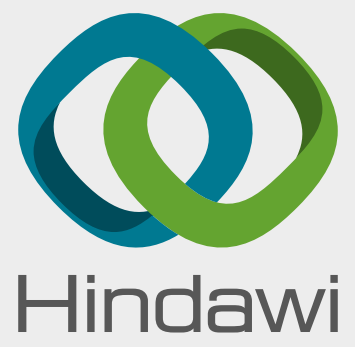

Submit your manuscripts at

www.hindawi.com
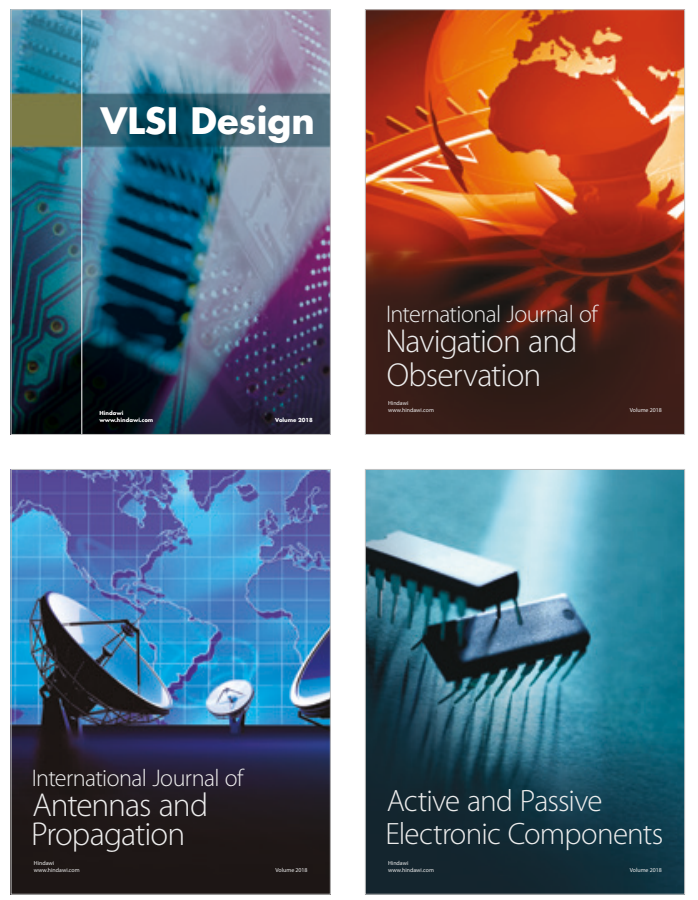
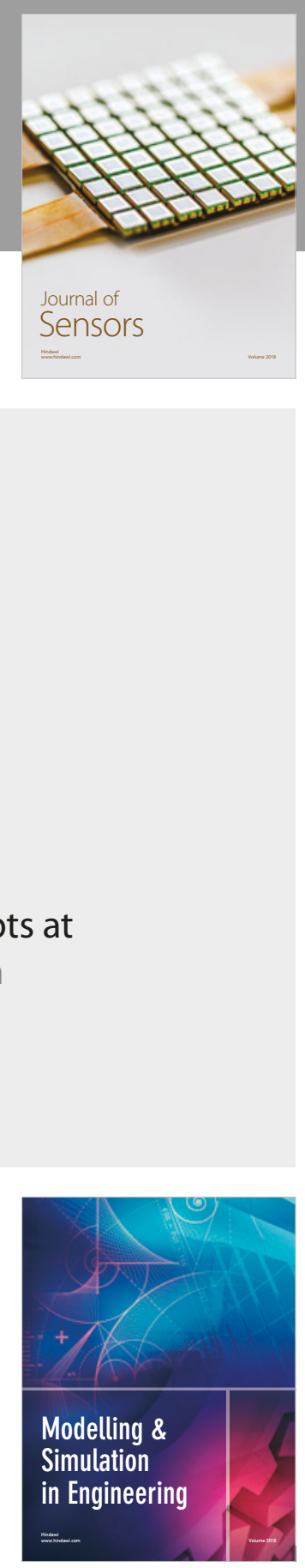

\section{Advances \\ Multimedia}
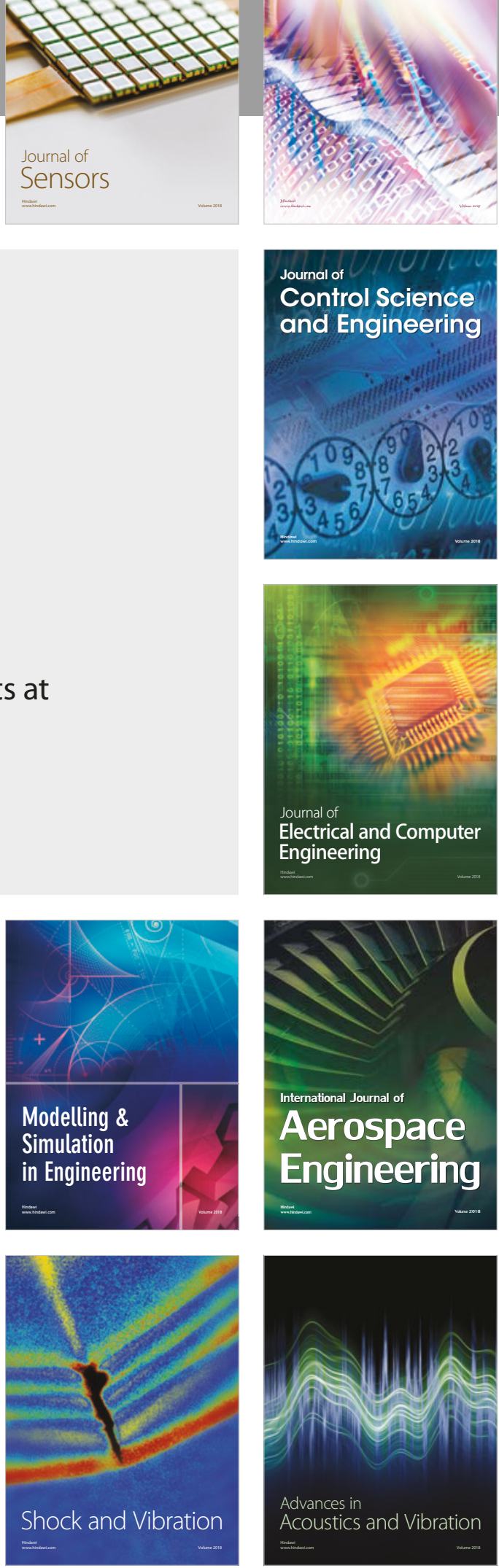